\title{
Mohammad Saleh
}

On weakly projective and weakly injective modules

Commentationes Mathematicae Universitatis Carolinae, Vol. 45 (2004), No. 3, 389--402

Persistent URL: http://dml.cz/dmlcz/119468

\section{Terms of use:}

(C) Charles University in Prague, Faculty of Mathematics and Physics, 2004

Institute of Mathematics of the Academy of Sciences of the Czech Republic provides access to digitized documents strictly for personal use. Each copy of any part of this document must contain these Terms of use.

This paper has been digitized, optimized for electronic delivery and stamped
with digital signature within the project $D M L-C Z$ : The Czech Digital
Mathematics Library http://project.dml.cz




\title{
On weakly projective and weakly injective modules
}

\author{
Mohammad Saleh
}

\begin{abstract}
The purpose of this paper is to further the study of weakly injective and weakly projective modules as a generalization of injective and projective modules. For a locally q.f.d. module $M$, there exists a module $K \in \sigma[M]$ such that $K \oplus N$ is weakly injective in $\sigma[M]$, for any $N \in \sigma[M]$. Similarly, if $M$ is projective and right perfect in $\sigma[M]$, then there exists a module $K \in \sigma[M]$ such that $K \oplus N$ is weakly projective in $\sigma[M]$, for any $N \in \sigma[M]$. Consequently, over a right perfect ring every module is a direct summand of a weakly projective module. For some classes $\mathcal{M}$ of modules in $\sigma[M]$, we study when direct sums of modules from $\mathcal{M}$ satisfy property $\mathbb{P}$ in $\sigma[M]$. In particular, we get characterizations of locally countably thick modules, a generalization of locally q.f.d. modules.
\end{abstract}

Keywords: tight, weakly tight, weakly injective, weakly projective, countably thick, locally q.f.d., weakly semisimple

Classification: 16D50, 16D60, 16D70, 16P40

\section{Introduction}

Throughout this paper all rings are associative with identity and all modules are unitary. We denote the category of all right $R$-modules by Mod- $R$ and for any $M \in \operatorname{Mod}-R, \sigma[M]$ stands for the full subcategory of Mod- $R$ whose objects are submodules of $M$-generated modules (see [29]). Given a module $X_{R}$, the injective hull of $X$ in Mod- $R$ (resp., in $\sigma[M]$ ) is denoted by $E(X)$ (resp., $\widehat{X}$ ). The $M$-injective hull $\widehat{X}$ is the trace of $M$ in $E(X)$, i.e. $\widehat{X}=\sum\{f(M), f \in$ $\operatorname{Hom}(M, E(X))\}$.

The purpose of this paper is to further the study of the concepts of weak injectivity (projectivity) in $\sigma[M]$ studied in [4], [9], [21], [24], [25], [26], [27], [30], [31]. In view of Theorem 2.9, if a right module $M$ is projective and right perfect in $\sigma[M]$, then there exists a module $K \in \sigma[M]$ such that $K \oplus X$ is a weakly projective module, for every module $X \in \sigma[M]$. Consequently, over a right perfect ring every module is a direct summand of a weakly projective module which was proved by S.K. Jain, S.R. López-Permouth and M. Saleh. Similarly, every module $X$ in $\sigma[M]$ is a direct summand of a weakly injective module in $\sigma[M]$, a result that generalizes $2.12,2.13$ and 2.14 in [17], 2.1, 2.2. and 2.3 in [19]. For a locally q.f.d. module $M$, there exists a module $K \in \sigma[M]$ such that $K \oplus N$ is weakly injective in $\sigma[M]$, for any $N \in \sigma[M]$. For some classes $\mathcal{M}$ of modules in $\sigma[M]$ 
we study when direct sums of modules from $\mathcal{M}$ are weakly tight in $\sigma[M]$. In particular, we get necessary and sufficient conditions for $\sum$-weak tightness of the injective hull of a simple module. As a consequence, we get characterizations of q.f.d. rings by means of weakly injective (tight) modules given by A. Al-Huzali, S.K. Jain and S.R. López-Permouth.

Given two modules $Q$ and $N \in \sigma[M]$, we call $Q$ weakly $N$-injective in $\sigma[M]$ if for every homomorphism $\varphi: N \rightarrow \widehat{Q}$, there exists a homomorphism $\widehat{\varphi}: N \rightarrow Q$ and a monomorphism $\sigma: Q \rightarrow \widehat{Q}$ such that $\varphi=\sigma \widehat{\varphi}$. Equivalently, there exists a submodule $X$ of $\widehat{Q}$ such that $\varphi(N) \subset X \simeq Q$. A module $Q \in \sigma[M]$ is called weakly injective in $\sigma[M]$ if for every finitely generated submodule $N$ of the $M$-injective hull $\widehat{Q}, N$ is contained in a submodule $Y$ of $\widehat{Q}$ such that $Y \simeq Q$. Equivalently, if $Q$ is weakly $N$-injective for all finitely generated modules $N$ in $\sigma[M]$. A module $X$ is $N$-tight in $\sigma[M]$ if every quotient of $N$ which is embeddable in the $M$-injective hull of $X$ is embeddable in $X$. A module is tight (R-tight) in $\sigma[M]$ if it is tight relative to all finitely generated (cyclic) submodules of its $M$-injective hull, and $Q$ is weakly tight (weakly R-tight) in $\sigma[M]$ if every finitely generated (cyclic) submodule $N$ of $\widehat{Q}$ is embeddable in a direct sum of copies of $Q$. It is clear that every weakly injective module in $\sigma[M]$ is tight in $\sigma[M]$, and every tight module in $\sigma[M]$ is weakly tight in $\sigma[M]$, but weak tightness does not imply tightness (see $[4],[31])$.

Given two modules $Q, N \in \sigma[M]$, we call $Q$ weakly $N$-projective in $\sigma[M]$ if $Q$ has a $\sigma[M]$-projective cover $P(Q)$ (see [29, Section 19.4]) and for every homomorphism $\varphi: P(Q) \rightarrow N$ there exists a homomorphism $\widehat{\varphi}: Q \rightarrow N$ and an epimorphism $\sigma: P(Q) \rightarrow Q$ such that $\varphi=\widehat{\varphi} \sigma$. Equivalently, $Q$ is weakly $N$-projective in $\sigma[M]$ if for every homomorphism $\varphi: P(Q) \rightarrow N$, there exists a submodule $X$ of $\operatorname{ker}(\varphi)$ such that $P(Q) / X \simeq Q$. A module $Q \in \sigma[M]$ is called weakly projective in $\sigma[M]$ if it is weakly $N$-projective for all finitely $M$-generated modules $N$ in $\sigma[M]$. A module $M_{R}$ is called locally q.f.d. ([3], [7], [18]) if every finitely generated (or cyclic) module $N \in \sigma[M]$ has finite uniform dimension. For a right $R$-module and $N$ in $\sigma[M], N$ is called perfect in $\sigma[M]$ if for any index set $\Lambda$, the sum $N^{(\Lambda)}$ is semiperfect in $\sigma[M]$ (see [29, Section 43]). A module $Q$ is called weakly $(N$-)projective (resp., weakly $(N$-)injective, tight) $([17],[14],[15],[16])$ if it is weakly $\left(N\right.$-)projective (resp., weakly $\left(N\right.$-)injective, tight) in $\sigma\left[R_{R}\right]=\operatorname{Mod}-R$.

\section{A large class of modules}

The class of weakly injective (tight, weakly tight) modules in $\sigma[M]$ is closed under finite direct sums, and essential extensions, and the class of weakly projective modules in $\sigma[M]$ is closed under finite direct sums. Also, the domains of the class of weakly injective (tight, weakly tight, weakly projective) modules in $\sigma[M]$ are closed under submodules and quotients.

First, we list below some known results on weak projectivity and weak injec- 
tivity (tightness) in $\sigma[M]$ that will be needed through this paper (cf. [4], [24], [23], [25], [30], [31]).

Lemma 2.1 ([24, Lemma 2.2]). Let $\left\{X_{i}\right\}_{I}$ be a class of weakly $N$-projective modules in $\sigma[M]$ and let $\bigoplus_{I} X_{i}$ have a projective cover in $\sigma[M]$. Then $\bigoplus_{I} X_{i}$ is weakly $N$-projective in $\sigma[M]$.

Proof: The proof follows directly from the fact that in this case $P\left(\bigoplus_{I} X_{i}\right)=$ $\bigoplus_{I} P\left(X_{i}\right)$.

Lemma 2.2. Given modules $N, Q \in \sigma[M]$, the following hold true.

(a) If $Q$ is self-projective and weakly $N$-projective in $\sigma[M]$, then $Q$ is $N$ projective in $\sigma[M]$.

(b) If $Q$ is self-injective and $N$-tight in $\sigma[M]$, then $Q$ is $N$-injective in $\sigma[M]$.

Lemma 2.3. A finite direct sum of weakly injective (tight, weakly tight) modules in $\sigma[M]$ is weakly injective (tight, weakly tight) in $\sigma[M]$, and an essential extension of a weakly injective (tight, weakly tight) module in $\sigma[M]$ is weakly injective (tight, weakly tight) in $\sigma[M]$.

Lemma 2.4. A uniform module $X \in \sigma[M]$ is weakly tight in $\sigma[M]$ iff $X$ is weakly injective in $\sigma[M]$.

Proof: Let $X$ be uniform and weakly tight in $\sigma[M]$, and let $N$ be a finitely generated submodule of $\widehat{X}$. Then $N$ is embeddable in $X^{(\alpha)}$ via a monomorphism, say, $\phi$. Let $\pi_{i}: X^{(\alpha)} \rightarrow X$ be the $i$ th projection map. Then $\bigcap_{i \in \alpha} \operatorname{ker}\left(\pi_{i} \phi\right) \subseteq$ $\operatorname{ker} \phi=0$. Since $X$ is uniform, we have $\operatorname{ker}\left(\pi_{i} \phi\right)=0$, and thus $N$ embeds in $X$, proving that $X$ is tight. By Lemma $2.3, X$ is weakly injective in $\sigma[M]$.

Example 2.5. (i) [17, Example 2.11], [19]. Let $R$ be the ring of endomorphisms of an infinite dimensional vector space $V$ over a field $F$. Then $M=\operatorname{Soc}\left(R_{R}\right) \oplus R$ is tight but not weakly injective.

(ii) [4]. Let $R=Z$ and $X=(Q / Z) \oplus(Z / p Z)$. Then $X$ is weakly tight in $\sigma[M]$ but not tight.

(iii) $[17$, Example $4.4(\mathrm{~d})]$. Let $F$ be a field. Then $R=\left[\begin{array}{cc}F & F \\ 0 & F\end{array}\right]$ is weakly injective but the summand $S=\left[\begin{array}{ll}0 & 0 \\ 0 & F\end{array}\right]$ as an $R$-module is not weakly injective.

(iv) $[15$, Example 2.14(1)]. Let $R$ be a uniserial ring which is not a division ring (e.g. $Z /\left(p^{n}\right), p$ is prime), and $S=\operatorname{Soc}(R)$. Then, as a right $R$ module, $R / S \times R$ is weakly $R$-projective but not $R$-projective (see [14, Proposition 2.11]).

It has been shown in [17, Theorem 2.8] that if $\mathcal{K}$ is a class of modules that is closed under direct sums and under injective hulls, and if every cyclic module in $\mathcal{K}$ has finite uniform dimension, then every tight module in $\mathcal{K}$ is weakly injective. Taking $\mathcal{K}=\sigma[M]$, we obtain the following interesting corollary. 
Corollary 2.6. Let $M$ be a locally q.f.d. module. Then every tight module in $\sigma[M]$ is weakly injective in $\sigma[M]$.

Theorem 2.7. Let $M$ be a locally q.f.d. module. Then there exists a module $K \in \sigma[M]$ such that $Q=K \oplus N$ is a weakly injective module for every module $N \in \sigma[M]$.

ProOF: Let $\mathcal{F}$ be the family of all indecomposable injectives up to isomorphism in $\sigma[M]$ and let $K=\bigoplus \sum_{F \in \mathcal{F}} F^{(\alpha)}$ where $\alpha$ is an infinite cardinal number greater than both the cardinality of $M$ and the cardinality of the ring $R$. Let $Q=K \oplus N$. Then $Q$ is weakly injective in $\sigma[M]$ for every module $N \in \sigma[M]$, since every finitely generated module over a locally q.f.d. module is embeddable in a finite direct sum of indecomposable injectives and thus embeddable in $Q$. Thus $Q$ is tight in $\sigma[M]$ and thus, $Q$ is weakly injective in $\sigma[M]$.

In [19], it is shown that any semisimple module is a direct summand of a weakly injective module. The next lemma shows that in fact any module is a direct summand of a weakly injective module.

Lemma 2.8 ([26, Lemma 2.3]). Every module in $\sigma[M]$ is a direct summand of a weakly injective module in $\sigma[M]$.

Proof: Follows from the fact that for any module $X$ in $\sigma[M], X \oplus \widehat{(X)^{(\alpha)}}$ is weakly injective in $\sigma[M]$ where $\alpha$ is an infinite cardinal number.

The above result generalizes $2.12,2.13$ and 2.14 in [17], and 2.1, 2.2. and 2.3 in [19].

The next is a dual to Lemma 2.8 for weak projectivity.

Theorem 2.9. Let $M$ be projective and right perfect in $\sigma[M]$. Then there exists a module $K \in \sigma[M]$ such that $K \oplus X$ is a weakly projective module in $\sigma[M]$ for every module $X \in \sigma[M]$.

Proof: Let $L$ be the direct sum of all finitely $M$-generated modules (up to isomorphism) in $\sigma[M]$. Let $K=L \oplus[P(L)]^{(\alpha)}$ where $\alpha$ is an infinite cardinal number greater than both the cardinality of $M$ and the cardinality of the ring $R$. We claim that $Q=X \oplus K$ is weakly projective in $\sigma[M]$ for every module $X \in \sigma[M]$. Let $\varphi: P(Q) \rightarrow N$ be an epimorphism, where $N$ is a finitely $M$ generated module in $\sigma[M]$. Let $\pi: P(N) \rightarrow N$ be the $M$-projective cover map. By the projectivity of $P(Q)$, there exists a homomorphism $\widehat{\varphi}: P(Q) \rightarrow P(N)$ such that $\pi \widehat{\varphi}=\varphi$. Since $\operatorname{Ker} \pi \ll P(N), \widehat{\varphi}$ is onto. Since $P(N)$ is projective, $\widehat{\varphi}$ splits, and therefore we may write $P(Q)=P \oplus \operatorname{Ker} \widehat{\varphi}$, for some submodule $P \subset P(Q)$ isomorphic to $P(N)$. Also since $N$ is finitely $M$-generated, $P(N)$ is also finitely M-generated and thus $P(N)$ is a direct summand of $P(Q)$. Since $M$ is perfect, $M \cong \bigoplus_{\lambda=1}^{n} L_{\lambda}$, where each $L_{\lambda}$ is local. It follows by [29, Section 41.17] that every projective module in $\sigma[M]$ is a projective cover of a simple module 
and thus a direct sum of the indecomposable local projective modules $L_{\lambda}$ in $\sigma[M]$. Write $P(N) \cong \bigoplus_{\lambda=1}^{n} L_{\lambda}^{\alpha_{i}}$, Ker $\widehat{\varphi} \cong \bigoplus_{\lambda=1}^{n} L_{\lambda}^{\beta_{i}}, P(L) \cong \bigoplus_{\lambda=1}^{n} L_{\lambda}^{\gamma_{i}}$, and $P(Q) \cong \bigoplus_{\lambda=1}^{n} L_{\lambda}^{\lambda_{i}}$. Since $P(N)$ is finitely generated, $\alpha_{i}$ are finite. Now, it follows easily that $P(Q) \cong \operatorname{Ker} \widehat{\varphi}$, and one may think of $\widehat{\varphi}$ as the projection map $p: P(Q) \oplus P(N) \rightarrow P(N)$. It follows that $\operatorname{Ker} \varphi \cong P(Q) \oplus \operatorname{Ker} \pi$. Now $Q$ is a homomorphic image of $P(Q)$ and, by definition of $L$, there exists a submodule $Q^{\prime} \subset Q$ such that $N \oplus Q^{\prime}=Q$. Thus there exists a submodule $K^{\prime} \subset P(Q)$ such that $P(Q) / K^{\prime} \cong Q^{\prime}$. Let $X=K^{\prime} \oplus \operatorname{Ker} \pi \subset \operatorname{Ker} \varphi$. Then $P(Q) / X=$ $[P(Q) \oplus P(N)] /\left[K^{\prime} \oplus \operatorname{Ker} \pi\right] \cong Q^{\prime} \oplus N \cong Q$, as desired.

The above results show that the classes of weakly injective and weakly projective modules are quite large.

We call a module $M_{R}$ weakly semisimple (weakly $R$-semisimple) iff every module $N \in \sigma[M]$ is weakly injective (weakly $R$-injective) in $\sigma[M]$. As a direct application of the above results, we state the following characterizations of semisimple and weakly $(R$-)semisimple modules in terms of weak injectivity, tightness, weak tightness, and weak projectivity. The proofs are straightforward, for the sake of convenience of the reader we provide proofs to some of these implications.

Theorem 2.10. For a module $M_{R}$, the following are equivalent:

(a) $M$ is semisimple;

(b) $M$ is projective and perfect in $\sigma[M]$ and every weakly projective module in $\sigma[M]$ is (quasi-) discrete;

(c) $M$ is projective and perfect in $\sigma[M]$ and every discrete module is weakly projective in $\sigma[M]$;

(d) $M$ is projective and perfect in $\sigma[M]$ and every weakly projective module in $\sigma[M]$ is (quasi-)continuous;

(e) every weakly injective module in $\sigma[M]$ is (quasi-)discrete;

(f) every weakly injective module in $\sigma[M]$ is (quasi-)continuous;

(g) every continuous module is weakly projective in $\sigma[M]$;

(h) every (direct summand of a) weakly injective module in $\sigma[M]$ is (injective) projective in $\sigma[M]$;

(i) $M$ is projective and perfect and every weakly projective module in $\sigma[M]$ is injective (projective) in $\sigma[M]$;

(j) $M$ is projective and perfect in $\sigma[M]$ and every direct summand of a weakly projective module in $\sigma[M]$ is weakly projective in $\sigma[M]$;

(k) $M$ is projective and perfect in $\sigma[M]$ and every (direct summand of a) weakly projective module in $\sigma[M]$ is quasi-projective in $\sigma[M]$;

(l) every direct summand of a weakly injective module in $\sigma[M]$ is quasiinjective in $\sigma[M]$;

(m) $M$ is projective and perfect in $\sigma[M]$ and every direct summand of a weakly projective module in $\sigma[M]$ is injective in $\sigma[M]$. 
Proof: $(\mathrm{h}) \Rightarrow(\mathrm{a})$. Let $X \in \sigma[M]$. By Theorem 2.8, $X \oplus \widehat{(X)^{(\alpha)}}$ is weakly injective in $\sigma[M]$, where $\alpha$ is an infinite cardinal number. Thus $X$ is injective, proving that $M$ is semisimple.

(i) $\Rightarrow(\mathrm{a})$. Let $N \in \sigma[M]$. By Theorem 2.9, there exists a module $Q \in \sigma[M]$ such that $Q \oplus N$ is weakly projective and thus $Q \oplus N$ is injective, and thus $N$ is injective, proving that $M$ is semisimple.

Clearly (a) implies all other items. The other implications are similar and thus are left to the reader.

Theorem 2.11. For a module $M_{R}$, the following are equivalent:

(a) $M$ is weakly semisimple (resp., weakly $R$-semisimple);

(b) $M$ is projective and perfect in $\sigma[M]$ and every direct summand of a weakly projective module in $\sigma[M]$ is weakly injective (or tight, weakly tight) (resp., weakly $R$-injective) (or $R$-tight, weakly $R$-tight) in $\sigma[M]$;

(c) every direct summand of a weakly injective (or tight, weakly tight) (resp., weakly $R$-injective) (or $R$-tight, weakly $R$-tight) module in $\sigma[M]$ is weakly injective (or tight, weakly tight) (resp., weakly $R$-injective) (or $R$-tight, weakly $R$-tight) in $\sigma[M]$.

Proof: $(\mathrm{b}) \Rightarrow(\mathrm{a})$. Let $N \in \sigma[M]$. By Theorem 2.9, there exists a module $Q \in \sigma[M]$ such that $Q \oplus N$ is weakly projective and thus $N$ is weakly injective, proving that $M$ is weakly semisimple.

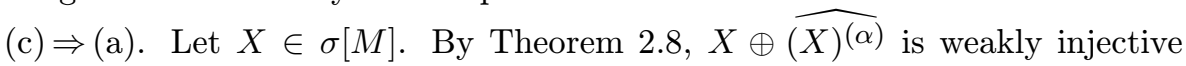
in $\sigma[M]$, where $\alpha$ is an infinite cardinal number. Thus $X$ is weakly injective, proving that $M$ is weakly semisimple. The other cases are similar and are left to the reader.

In case $M=R$ in the above two theorems we get the following characterization of semisimple, weakly semisimple, and weakly $R$-semisimple rings.

Corollary 2.12. For a ring $R$, the following are equivalent:

(a) $R$ is semisimple;

(b) $R$ is perfect and every weakly projective module is (quasi-)discrete;

(c) $R$ is perfect and every discrete module is weakly projective;

(d) $R$ is perfect and every weakly projective module is (quasi-)continuous;

(e) every weakly injective module is (quasi-)discrete;

(f) every weakly injective module is (quasi-)continuous;

(g) every continuous module is weakly projective;

(h) every (direct summand of a) weakly injective module is (injective) projective;

(i) $R$ is perfect and every weakly projective module is injective (projective);

(j) $R$ is perfect and every direct summand of a weakly projective module is weakly projective; 
(k) $R$ is perfect and every (direct summand of a) weakly projective module is quasi-projective;

(l) every direct summand of a weakly injective module is quasi-injective;

(m) $R$ is perfect and every direct summand of a weakly projective module is injective.

Corollary 2.13. For a ring $R$, the following are equivalent:

(a) $R$ is weakly semisimple (resp., weakly $R$-semisimple);

(b) $R$ is perfect and every direct summand of a weakly projective module is weakly injective (or tight, weakly tight) (resp., weakly $R$-injective) (or $R$-tight, weakly $R$-tight);

(c) every direct summand of a weakly injective (or tight, weakly tight) (resp., weakly $R$-injective) (or $R$-tight, weakly $R$-tight) module is weakly injective (or tight, weakly tight) (resp., weakly $R$-injective) (or $R$-tight, weakly $R$ tight).

\section{Direct sums of classes of modules}

Let $M_{R}$ be a fixed module and $\mathcal{K}$ a class of simple modules in $\sigma[M]$. We denote

$$
\operatorname{Soc}_{\mathcal{K}}(X)=\sum\{A \subseteq X \mid A \simeq P \text { for some } P \in \mathcal{K}\}
$$

Recall ([4], [5], [6]) that $X \in \sigma[M]$ is said to be countably thick relative to $\mathcal{K}$ if $\operatorname{Soc}_{\mathcal{K}}(X / A)$ is finitely generated for all $A \subseteq X$. In particular, if $\mathcal{K}$ is the class of all simple modules in $\sigma[M]$ then $X \in \sigma[M]$ is countably thick relative to $\mathcal{K}$ if and only if all factor modules of $X$ have finite uniform dimension, that is $X$ is q.f.d. (see [4, Lemma 1], [5], [6]).

For a module $X_{R}$ and a module property $\mathbb{P}, X$ is said to be $\sum-\mathbb{P}$ in case every direct sum of copies of $X$ enjoys the property $\mathbb{P}$. Also we call $X$ locally $\mathbb{P}$ in case every finitely generated submodule of $X$ enjoys the property $\mathbb{P}$ (see [1], [3], [18]).

Theorem 3.1. For a module $M_{R}$, the following implications $(\mathrm{a}) \Rightarrow(\mathrm{b}) \Rightarrow(\mathrm{c}) \Rightarrow$ $(\mathrm{d}) \Rightarrow(\mathrm{e}) \Rightarrow(\mathrm{f})$ always hold:

(a) every direct sum $\bigoplus_{\Lambda} E_{\lambda}$ of injectives in $\sigma[M]$, where each $E_{\lambda}$ is essential over $\operatorname{Soc}_{\mathcal{K}}\left(E_{\lambda}\right)$, is weakly injective in $\sigma[M]$;

(b) every direct sum $\bigoplus_{\Lambda} M_{\lambda}$ of weakly injective modules in $\sigma[M]$, where each $M_{\lambda}$ is essential over $\operatorname{Soc}_{\mathcal{K}}\left(M_{\lambda}\right)$, is weakly injective in $\sigma[M]$;

(c) every direct sum $\bigoplus_{\Lambda} M_{\lambda}$ of weakly injective modules in $\sigma[M]$, where each $M_{\lambda}$ is essential over $\operatorname{Soc}_{\mathcal{K}}\left(M_{\lambda}\right)$, is tight in $\sigma[M]$;

(d) every direct sum $\bigoplus_{\Lambda} M_{\lambda}$ of tight modules in $\sigma[M]$, where each $M_{\lambda}$ is essential over $\operatorname{Soc}_{\mathcal{K}}\left(M_{\lambda}\right)$, is tight in $\sigma[M]$;

(e) every direct sum $\bigoplus_{\Lambda} M_{\lambda}$ of tight modules in $\sigma[M]$, where each $M_{\lambda}$ is essential over $\operatorname{Soc}_{\mathcal{K}}\left(M_{\lambda}\right)$, is weakly tight in $\sigma[M]$; 
(f) every direct sum $\bigoplus_{\Lambda} M_{\lambda}$ of weakly tight modules in $\sigma[M]$, where each $M_{\lambda}$ is essential over $\operatorname{Soc}_{\mathcal{K}}\left(M_{\lambda}\right)$, is weakly tight in $\sigma[M]$.

Proof: $(\mathrm{a}) \Rightarrow(\mathrm{b})$. Consider the module $X=\bigoplus_{\Lambda} M_{\lambda}$, a direct sum of weakly injective modules in $\sigma[M]$, where each $M_{\lambda}$ is essential over $\operatorname{Soc}_{\mathcal{K}}\left(M_{\lambda}\right)$. Let $N$ be a finitely generated submodule of $\widehat{X}$. By (a) the direct sum $\bigoplus_{\Lambda} \widehat{M_{\lambda}}$ is weakly injective in $\sigma[M]$ and $X=\bigoplus_{\Lambda} M_{\lambda} \subseteq^{\prime} \bigoplus_{\Lambda} \widehat{M_{\lambda}} \subseteq^{\prime} \widehat{\bigoplus_{\Lambda} \widehat{M_{\lambda}}}$. Thus by (a) there exists a submodule $Y \subseteq \widehat{\bigoplus_{\Lambda} \widehat{M_{\lambda}}}$ such that $N \subseteq Y \cong \bigoplus_{\Lambda} \widehat{M}_{\lambda}$. Write $Y=\bigoplus_{\Lambda} \widehat{Y}_{\lambda}$, where $Y_{i} \cong M_{i}, i \in \Lambda$. Since $N$ is finitely generated, there exists a finite subset $\Gamma=\left\{\lambda_{1}, \ldots, \lambda_{m}\right\} \subseteq \Lambda$ such that $N \subseteq \bigoplus_{\Gamma} \widehat{Y_{\lambda}}=\widehat{\bigoplus_{\Gamma} Y_{\lambda}}$. Since $Y_{\lambda_{1}}, \ldots, Y_{\lambda_{m}}$ are weakly injective in $\sigma[M]$, the finite direct sum $Y_{\lambda_{1}} \oplus \cdots \oplus Y_{\lambda_{m}}$ is weakly injective in $\sigma[M]$. Therefore, there exists $X_{1} \cong \bigoplus_{\Gamma} Y_{\lambda} \cong \bigoplus_{\Gamma} M_{\lambda}$ such that $N \subseteq X_{1} \subseteq \widehat{\bigoplus_{\Gamma} Y_{\lambda}}$. Thus $N \subseteq X_{1} \oplus \bigoplus_{\lambda \notin \Gamma} Y_{\lambda} \simeq X$, proving that $X$ is weakly injective.

(c) $\Rightarrow$ (d). Consider the module $X=\bigoplus_{\Lambda} M_{\lambda}$ a direct sum of tight modules in $\sigma[M]$, where each $M_{\lambda}$ is essential over $\operatorname{Soc}_{\mathcal{K}}\left(M_{\lambda}\right)$. Let $N$ be a finitely generated submodule of $\widehat{X}=\widehat{\bigoplus_{\Lambda} \widehat{M_{\lambda}}}$. By (c), the direct sum $\bigoplus_{\Lambda} \widehat{M_{\lambda}}$ is tight in $\sigma[M]$. Thus $N$ embeds in $\bigoplus_{\Lambda} \widehat{M}_{\lambda}$ via a monomorphism, say, $\varphi$. Also $\varphi(N)$ is finitely generated and thus $N \subset \widehat{M}_{\lambda_{1}} \oplus \cdots \oplus \widehat{M}_{\lambda_{m}}=\bigoplus_{\lambda=1}^{m} \widehat{M_{\lambda}}$ for some finite $\left\{\lambda_{1}, \ldots, \lambda_{m}\right\} \subseteq \Lambda$. Since $M_{\lambda_{1}} \oplus \cdots \oplus M_{\lambda_{m}}$ is tight, $N \simeq \varphi(N)$ embeds in finite direct sums $M_{\lambda_{1}}$ $\oplus \cdots \oplus M_{\lambda_{m}}$, proving that $X$ is tight.

(e) $\Rightarrow$ (f). Consider the module $X=\bigoplus_{\Lambda} M_{\lambda}$, a direct sum of weakly tight modules in $\sigma[M]$, where each $M_{\lambda}$ is essential over $\operatorname{Soc}_{\mathcal{K}}\left(M_{\lambda}\right)$. Let $N$ be a finitely generated submodule of $\widehat{X}=\widehat{\bigoplus_{\Lambda} \widehat{M_{\lambda}}}$. By (e), the direct sum $\bigoplus_{\Lambda} \widehat{M_{\lambda}}$ is weakly tight in $\sigma[M]$. Thus $N$ embeds in $\left(\bigoplus_{\Lambda} \widehat{M}_{\lambda}\right)\left(\aleph_{0}\right)$ via a monomorphism, say, $\varphi$. Also $\varphi(N)$ is finitely generated and thus $N \subset \widehat{M}_{\lambda_{1}} \oplus \cdots \oplus \widehat{M}_{\lambda_{m}}=\bigoplus_{\lambda=1}^{m} \widehat{M_{\lambda}}$ for some finite $\left\{\lambda_{1}, \ldots, \lambda_{m}\right\} \subseteq \Lambda$. Since $M_{\lambda_{1}} \oplus \cdots \oplus M_{\lambda_{m}}$ is weakly tight, $N \simeq \varphi(N)$ embeds in a direct sums of copies of $\left(M_{\lambda_{1}} \oplus \cdots \oplus M_{\lambda_{m}}\right)$ and thus embeds in a direct sums of $X$, proving that $X$ is weakly tight.

Clearly, (b) $\Rightarrow(\mathrm{c})$ and $(\mathrm{d}) \Rightarrow(\mathrm{e})$.

The next theorem provides several characterizations of countably thick (consequently, locally q.f.d.) modules which extends the main result in [26]. Consequently, we get the main result in [2] as a corollary to the main results of this section.

Theorem 3.2. For a module $M_{R}$ and any class $\mathcal{K}$ of simple modules in $\sigma[M]$, the following conditions are equivalent:

(a) $M$ is locally countably thick relative to $\mathcal{K}$;

(b) every direct sum $\bigoplus_{\Lambda} E_{\lambda}$ of injectives in $\sigma[M]$, where each $E_{\lambda}$ is essential over $\operatorname{Soc}_{\mathcal{K}}\left(E_{\lambda}\right)$, is tight in $\sigma[M]$; 
(c) every direct sum $\bigoplus_{\Lambda} E_{\lambda}$ of tight modules in $\sigma[M]$, where each $E_{\lambda}$ is essential over $\operatorname{Soc}_{\mathcal{K}}\left(E_{\lambda}\right)$, is tight in $\sigma[M]$;

(d) every direct sum $\bigoplus_{\Lambda} E_{\lambda}$ of weakly tight modules in $\sigma[M]$, where each $E_{\lambda}$ is essential over $\operatorname{Soc}_{\mathcal{K}}\left(E_{\lambda}\right)$, is weakly tight in $\sigma[M]$;

(e) every direct sum $\bigoplus_{\Lambda} E_{\lambda}$ of weakly tight modules in $\sigma[M]$, where each $E_{\lambda}$ is essential over $\operatorname{Soc}_{\mathcal{K}}\left(E_{\lambda}\right)$, is weakly $N$-tight, for every cyclic module $N$ in $\sigma[M]$;

(f) every direct sum $\bigoplus_{\Lambda} \widehat{P_{\lambda}}$, where $P_{\lambda} \in \mathcal{K}$, is weakly $N$-tight, for every cyclic module $N$ in $\sigma[M]$.

Proof: (a) $\Rightarrow$ (b). Consider $X=\bigoplus_{\Lambda} E_{\lambda}$, where $E_{\lambda}$ is injective in $\sigma[M]$ for every $\lambda \in \Lambda$ and $\operatorname{Soc}_{\mathcal{K}}\left(E_{\lambda}\right)$ is essential in $E_{\lambda}$. Let $N$ be a finitely generated submodule of $\widehat{X}$. By the hypothesis, $\operatorname{Soc}_{\mathcal{K}}(N)$ is finitely generated that is

$\operatorname{Soc}_{\mathcal{K}}(N)=P_{1} \oplus \cdots \oplus P_{n}$ with $P_{i} \simeq P_{i}^{\prime}$ for some $P_{i}^{\prime} \in \mathcal{K}(1 \leq i \leq n)$.

$\operatorname{So}_{\operatorname{Soc}_{\mathcal{K}}}(N) \subseteq \operatorname{Soc}_{\mathcal{K}}(\widehat{X})=\operatorname{Soc}_{\mathcal{K}}(X) \subseteq X$ and hence $\operatorname{Soc}_{\mathcal{K}}(N) \subseteq E_{\lambda_{1}} \oplus \cdots \oplus E_{\lambda_{m}}$ for some finite $\left\{\lambda_{1}, \ldots, \lambda_{m}\right\} \subseteq \Lambda$. This implies that $E_{\lambda_{1}} \oplus \cdots \oplus E_{\lambda_{m}}$ contains an injective hull $E$ of $\operatorname{Soc}_{\mathcal{K}}(N)$. Thus $N$ embeds in $X$, proving that $X$ is tight.

(b) $\Rightarrow$ (c). Consider the module $X=\bigoplus_{\Lambda} M_{\lambda}$ a direct sum of tight modules in $\sigma[M]$, where each $M_{\lambda}$ is essential over $\operatorname{Soc}_{\mathcal{K}}\left(M_{\lambda}\right)$. Let $N$ be a finitely generated submodule of $\widehat{X}$. By (b), the direct sum $\bigoplus_{\Lambda} \widehat{M_{\lambda}}$ is tight in $\sigma[M]$ and thus $N$ embeds in $\bigoplus_{\Lambda} \widehat{M}_{\lambda}$ via a monomorphism, say, $\varphi$. Also $\varphi(N)$ is finitely generated and thus $N \subset \widehat{M}_{\lambda_{1}} \oplus \cdots \oplus \widehat{M}_{\lambda_{m}}$ for some finite $\left\{\lambda_{1}, \ldots, \lambda_{m}\right\} \subseteq \Lambda$. Since $M_{\lambda_{1}}$ $\oplus \cdots \oplus M_{\lambda_{m}}$ is tight, $N \simeq \varphi(N)$ embeds in $M_{\lambda_{1}} \oplus \cdots \oplus M_{\lambda_{m}}$ and thus embeds in $X$, proving that $X$ is tight.

(c) $\Rightarrow(\mathrm{d})$. Consider the module $X=\bigoplus_{\Lambda} M_{\lambda}$, a direct sum of weakly tight modules in $\sigma[M]$, where each $M_{\lambda}$ is essential over $\operatorname{Soc}_{\mathcal{K}}\left(M_{\lambda}\right)$. Let $N$ be a finitely generated submodule of $\widehat{X}$. By (c) the direct sum $\bigoplus_{\Lambda} \widehat{M}_{\lambda}$ is tight in $\sigma[M]$. Thus $N$ embeds in $\bigoplus_{\Lambda} \widehat{M}_{\lambda}$ via a monomorphism, say, $\varphi$. Also $\varphi(N)$ is finitely generated and thus $N \subset \widehat{M}_{\lambda_{1}} \oplus \cdots \oplus \widehat{M}_{\lambda_{m}}$ for some finite $\left\{\lambda_{1}, \ldots, \lambda_{m}\right\} \subseteq \Lambda$. Since $M_{\lambda_{1}}$ $\oplus \cdots \oplus M_{\lambda_{m}}$ is weakly tight, $N \simeq \varphi(N)$ embeds in a finite direct sums of $\left(M_{\lambda_{1}}\right.$ $\oplus \cdots \oplus M_{\lambda_{m}}$ ) and thus embeds in a finite direct sums of $X$, proving that $X$ is weakly tight.

Clearly, (d) $\Rightarrow(\mathrm{e}) \Rightarrow(\mathrm{f})$.

(f) $\Rightarrow$ (a). Let $K$ be a cyclic submodule of $M$. If $\operatorname{Soc}_{\mathcal{K}}(K)=0$, we are done. Suppose $0 \neq \operatorname{Soc}_{\mathcal{K}}(K)=\bigoplus_{\Lambda} P_{\lambda}$, where $P_{\lambda} \simeq P_{\lambda}^{\prime}$ for some $P_{\lambda}^{\prime} \in \mathcal{K}$. We shall show that $\operatorname{Soc}_{\mathcal{K}}(K)$ is finitely generated. To this end, consider the diagram

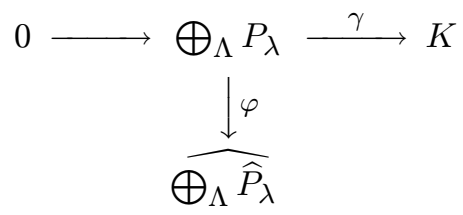


where $\varphi$ and $\gamma$ are the inclusion homomorphisms. By $M$-injectivity of $\widehat{\bigoplus_{\Lambda} \widehat{P}_{\lambda}}$, there exists $\psi: K \rightarrow \widehat{\bigoplus_{\Lambda} \widehat{P}_{\lambda}}$ such that $\psi \gamma=\varphi$. By our hypothesis, $\bigoplus_{\Lambda} \widehat{P}_{\lambda}$ is weakly $K$-tight in $\sigma[M]$, hence $\operatorname{Im} \varphi \subset \operatorname{Im} \psi$ is embeddable in $\left(\bigoplus_{\Lambda} \widehat{P}_{\lambda}\right)^{\left(\aleph_{0}\right)}$ and thus embeddable in a finite sum. Therefore, $\operatorname{Soc}_{\mathcal{K}}(K)$ is embeddable in $\widehat{P}_{\lambda_{1}} \oplus \cdots \oplus \widehat{P}_{\lambda_{m}}$ for some finite $\left\{\lambda_{1}, \ldots, \lambda_{m}\right\} \subseteq \Lambda$. Since each $\widehat{P}_{\lambda_{i}}$ is uniform, $\operatorname{Soc}_{\mathcal{K}}(K)$ has finite uniform dimension and is therefore finitely generated.

Combining Theorem 3.1 and Theorem 3.2 we get the following

Theorem 3.3. For a module $M_{R}$, and any class $\mathcal{K}$ of simple modules in $\sigma[M]$, the following conditions are equivalent:

(a) $M$ is locally countably thick relative to $\mathcal{K}$;

(b) every direct sum $\bigoplus_{\Lambda} E_{\lambda}$ of injectives in $\sigma[M]$, where each $E_{\lambda}$ is essential over $\operatorname{Soc}_{\mathcal{K}}\left(E_{\lambda}\right)$, is weakly injective in $\sigma[M]$;

(c) every direct sum $\bigoplus_{\Lambda} E_{\lambda}$ of injectives in $\sigma[M]$, where each $E_{\lambda}$ is essential over $\operatorname{Soc}_{\mathcal{K}}\left(E_{\lambda}\right)$, is tight in $\sigma[M]$;

(d) every direct sum $\bigoplus_{\Lambda} M_{\lambda}$ of weakly injective modules in $\sigma[M]$, where each $M_{\lambda}$ is essential over $\operatorname{Soc}_{\mathcal{K}}\left(M_{\lambda}\right)$, is weakly injective in $\sigma[M]$;

(e) every direct sum $\bigoplus_{\Lambda} M_{\lambda}$ of weakly injective modules in $\sigma[M]$, where each $M_{\lambda}$ is essential over $\operatorname{Soc}_{\mathcal{K}}\left(M_{\lambda}\right)$, is tight in $\sigma[M]$;

(f) every direct sum $\bigoplus_{\Lambda} M_{\lambda}$ of tight modules in $\sigma[M]$, where each $M_{\lambda}$ is essential over $\operatorname{Soc}_{\mathcal{K}}\left(M_{\lambda}\right)$, is tight in $\sigma[M]$;

(g) every direct sum $\bigoplus_{\Lambda} M_{\lambda}$ of tight modules in $\sigma[M]$, where each $M_{\lambda}$ is essential over $\operatorname{Soc}_{\mathcal{K}}\left(M_{\lambda}\right)$, is weakly tight in $\sigma[M]$;

(h) every direct sum $\bigoplus_{\Lambda} M_{\lambda}$ of weakly tight modules in $\sigma[M]$, where each $M_{\lambda}$ is essential over $\operatorname{Soc}_{\mathcal{K}}\left(M_{\lambda}\right)$, is weakly tight in $\sigma[M]$;

(i) every direct sum $\bigoplus_{\Lambda} M_{\lambda}$ of weakly tight modules in $\sigma[M]$, where each $M_{\lambda}$ is essential over $\operatorname{Soc}_{\mathcal{K}}\left(M_{\lambda}\right)$, is weakly $N$-tight, for every cyclic module $N$ in $\sigma[M]$;

(j) every direct sum $\bigoplus_{\Lambda} \widehat{P_{\lambda}}$, where $P_{\lambda} \in \mathcal{K}$, is weakly $N$-tight for every cyclic module $N$ in $\sigma[M]$

(k) every direct sum $\bigoplus_{\Lambda} \widehat{P_{\lambda}}$, where $P_{\lambda} \in \mathcal{K}$, is weakly $R$-tight in $\sigma[M]$.

Taking $\mathcal{K}$ to be all simple $R$-modules in $\sigma[M]$ in Theorem 3.3 we get $[26$, Theorem 2.7] as a corollary.

Corollary 3.4. For a module $M_{R}$, the following conditions are equivalent:

(a) $M$ is locally q.f.d.;

(b) every direct sum $\bigoplus_{\Lambda} E_{\lambda}$ of injectives in $\sigma[M]$ is weakly injective (tight, weakly tight) in $\sigma[M]$;

(c) every direct sum $\bigoplus_{\Lambda} E_{\lambda}$ of weakly injective in $\sigma[M]$ is weakly injective (tight, weakly tight) in $\sigma[M]$

(d) every direct sum of tight modules in $\sigma[M]$ is tight (weakly tight) in $\sigma[M]$; 
(e) every direct sum of weakly tight modules in $\sigma[M]$ is weakly tight (weakly $R$-tight) in $\sigma[M]$;

(f) every direct sum $\bigoplus_{\Lambda} \widehat{P_{\lambda}}$, where each $P_{\lambda}$ is simple, is weakly $N$-tight for every cyclic module $N$ in $\sigma[M]$;

(g) every direct sum $\bigoplus_{\Lambda} \widehat{P_{\lambda}}$, where each $P_{\lambda}$ is simple, is weakly $R$-tight in $\sigma[M]$.

In case $M=R_{R}$ in Corollary 3.4, we obtain characterizations of q.f.d. rings that generalize Theorem 2.6 and Corollary 2.7 in [30] and the main theorem in [2].

Corollary 3.5. For a ring $R$, the following conditions are equivalent:

(a) $R$ is q.f.d.;

(b) every direct sum $\bigoplus_{\Lambda} E_{\lambda}$ of injectives is weakly injective (or tight, weakly tight);

(c) every direct sum $\bigoplus_{\Lambda} E_{\lambda}$ of weakly injective is weakly injective (or tight, weakly tight);

(d) every direct sum of tight modules is tight (or weakly tight);

(e) every direct sum of weakly tight module is weakly tight (or weakly $R$ tight);

(f) every direct sum $\bigoplus_{\Lambda} E\left(P_{\lambda}\right)$, where each $P_{\lambda}$ is simple, is weakly $N$-tight for every cyclic module $N$;

(g) every direct sum $\bigoplus_{\Lambda} E\left(P_{\lambda}\right)$, where each $P_{\lambda}$ is simple, is weakly $R$-tight.

Theorem 3.6. A locally right q.f.d. module $M_{R}$ over which every uniform cyclic right module in $\sigma[M]$ is weakly injective (tight, weakly tight) in $\sigma[M]$ is right weakly semisimple.

Proof: Let $N \in \sigma[M]$. Then $N$ contains an essential submodule $X=\bigoplus_{I} X_{i}$ which is a direct sum of cyclic uniform submodules. It follows by our hypothesis that each $X_{i}$ is weakly injective in $\sigma[M]$ and thus by Lemma $2.3, \bigoplus_{I} X_{i}$ is weakly injective in $\sigma[M]$. Thus $N$ is weakly injective in $\sigma[M]$, proving that $M$ is weakly semisimple.

Theorem 3.7. For a module $M_{R}$, the following conditions are equivalent:

(a) $M$ is weakly semisimple;

(b) $M$ is locally q.f.d. and every finitely generated module in $\sigma[M]$ is weakly injective (tight, weakly tight) in $\sigma[M]$;

(c) $M$ is locally q.f.d. and every cyclic module in $\sigma[M]$ is weakly injective (tight, weakly tight) in $\sigma[M]$

(d) $M$ is locally q.f.d. and every uniform cyclic module in $\sigma[M]$ is weakly injective (tight, weakly tight) in $\sigma[M]$;

(e) $M$ is locally q.f.d. and every finitely generated module in $\sigma[M]$ is compressible. 
Proof: $(\mathrm{a}) \Rightarrow(\mathrm{b})$. Follows from Corollary 3.5.

Clearly, (b) $\Rightarrow$ (c) $\Rightarrow$ (d).

(d) $\Rightarrow$ (e). Let $N$ be a finitely generated module in $\sigma[M]$ and let $K \subseteq^{\prime} N$. Since $M$ is locally q.f.d., $N$ has finite uniform dimension. Thus there exist cyclic uniform submodules $U_{i}, i=1, \ldots, n$, of $N$ such that $\bigoplus_{i=1}^{i=n} U_{i} \subseteq^{\prime} K \subseteq N$. Since each $U_{i}$ is uniform it follows that each $U_{i}$ is weakly injective in $\sigma[M]$ and thus by Lemma 2.4, $\bigoplus_{i=1}^{i=n} U_{i}$ is weakly injective in $\sigma[M]$. Thus, by Lemma $2.3, K$ is weakly injective in $\sigma[M]$ and thus $N$ embeds in $K$, proving that $N$ is compressible.

(e) $\Rightarrow($ a). Let $0 \neq X$ in $\sigma[M]$ and let $N$ be a finitely generated submodule of $\widehat{X}$. Let $0 \neq x \in X$. Then $x R \cap N \subseteq^{\prime} N$. By our hypothesis $N$ is compressible and thus $N$ embeds in $x R \cap N$ and thus embeds in $X$, proving that $X$ is tight in $\sigma[M]$. Thus, $M$ is weakly semisimple by Theorem 3.6.

As a consequence of Theorem 3.7 we get [9, Theorem 3.1].

In case $M=R$ we obtain characterizations of weakly semisimple rings that generalize those known results.

Corollary 3.8. For a ring $R$, the following conditions are equivalent:

(a) $R$ is weakly semisimple;

(b) $R$ is q.f.d. and every finitely generated module is weakly injective (tight, weakly tight);

(c) $R$ is q.f.d. and every cyclic module is weakly injective (tight, weakly tight);

(d) $R$ is q.f.d. and every uniform cyclic module is weakly injective (tight, weakly tight);

(e) $R$ is q.f.d. and every finitely generated module is compressible.

We conclude with the following open questions:

(1) Can we replace the assumption of perfectness of a ring in Theorem 2.9 by semiperfectness?

(2) Can we remove locally q.f.d. throughout Theorem 3.7 and in Theorem 3.6?

Acknowledgment. The author wishes to thank the learned referee for his valuable comments that improved the writing of this paper. A part of this paper was written during the stay of the author at Ohio University under a Fulbright scholarship. The author wishes to thank the Department of Mathematics and the Ohio University Center for Ring Theory and its Applications for the warm hospitality and the Fulbright for the financial support.

\section{REFERENCES}

[1] Albu T., Nastasescu C., Relative Finiteness in Module Theory, Marcel Dekker, 1984.

[2] Al-Huzali A., Jain S.K., López-Permouth S.R., Rings whose cyclics have finite Goldie dimension, J. Algebra 153 (1992), 37-40.

[3] Berry D., Modules whose cyclic submodules have finite dimension, Canad. Math. Bull. 19 (1976), 1-6. 
[4] Brodskii G., Saleh M., Thuyet L., Wisbauer R., On weak injectivity of direct sums of modules, Vietnam J. Math. 26 (1998), 121-127.

[5] Brodskii G., Denumerable distributivity, linear compactness and the AB5* condition in modules, Russian Acad. Sci. Dokl. Math. 53 (1996), 76-77.

[6] Brodskii G., The Grothendieck condition AB $5^{*}$ and generalizations of module distributivity, Russ. Math. 41 (1997), 1-11.

[7] Camillo V.P., Modules whose quotients have finite Goldie dimension, Pacific J. Math. 69 (1977), 337-338.

[8] Dung N.V., Huynh D.V., Smith P.F., Wisbauer R., Extending Modules, Pitman, London, 1994.

[9] Dhompong S., Sanwong J., Plubtieng S., Tansee H., On modules whose singular subgenerated modules are weakly injective, Algebra Colloq. 8 (2001), 227-236.

[10] Goel V.K., Jain S.K., $\pi$-injective modules and rings whose cyclic modules are $\pi$-injective, Comm. Algebra 6 (1978), 59-73.

[11] Golan J.S., López-Permouth S.R., QI-filters and tight modules, Comm. Algebra 19 (1991), $2217-2229$.

[12] Jain S.K., López-Permouth S.R., Rings whose cyclics are essentially embeddable in projective modules, J. Algebra 128 (1990), 257-269.

[13] Jain S.K., López-Permouth S.R., Risvi T., A characterization of uniserial rings via continuous and discrete modules, J. Austral. Math. Soc., Ser. A 50 (1991), 197-203.

[14] Jain S.K., López-Permouth S.R., Saleh M., On weakly projective modules, in: Ring Theory, Proceedings, OSU-Denison conference 1992, World Scientific Press, New Jersey, 1993, pp. 200-208.

[15] Jain S.K., López-Permouth S.R., Oshiro K., Saleh M., Weakly projective and weakly injective modules, Canad. J. Math. 34 (1994), 972-981.

[16] Jain S.K., López-Permouth S.R., Singh S., On a class of QI-rings, Glasgow J. Math. 34 (1992), 75-81.

[17] Jain S.K., López-Permouth S.R., A survey on the theory of weakly injective modules, in: Computational Algebra, Lecture Notes in Pure and Applied Mathematics, Marcel Dekker, Inc., New York, 1994, pp. 205-233.

[18] Kurshan A.P., Rings whose cyclic modules have finitely generated socle, J. Algebra 14 (1970), 376-386.

[19] López-Permouth S.R., Rings characterized by their weakly injective modules, Glasgow Math. J. 34 (1992), 349-353.

[20] Malik S., Vanaja N., Weak relative injective $M$-subgenerated modules, Advances in Ring Theory, Birkhäuser, Boston, 1997, pp. 221-239.

[21] Mohamed S., Muller B., Singh S., Quasi-dual continuous modules, J. Austral. Math. Soc., Ser. A 39 (1985), 287-299.

[22] Mohamed S., Muller B,, Continuous and Discrete Modules, Cambridge University Press, 1990.

[23] Saleh M., A note on tightness, Glasgow Math. J. 41 (1999), 43-44.

[24] Saleh M., Abdel-Mohsen A., On weak injectivity and weak projectivity, in: Proceedings of the Mathematics Conference, World Scientific Press, New Jersey, 2000, pp. 196-207.

[25] Saleh M., Abdel-Mohsen A., A note on weak injectivity, Far East J. Math. Sci. 11 (2003), 199-206.

[26] Saleh M., On q.f.d. modules and q.f.d. rings, Houston J. Math., to appear.

[27] Sanh N.V., Shum K.P., Dhompongsa S., Wongwai S., On quasi-principally injective modules, Algebra Colloq. 6 (1999), 296-276.

[28] Sanh N.V., Dhompongsa S., Wongwai S., On generalized q.f.d. modules and rings, Algebra and Combinatorics, Springer-Verlag, 1999, pp. 367-272.

[29] Wisbauer R., Foundations of Module and Ring Theory, Gordon and Breach, 1991. 
[30] Zhou Y., Notes on weakly semisimple rings, Bull. Austral. Math. Soc. 52 (1996), 517-525.

[31] Zhou Y., Weak injectivity and module classes, Comm. Algebra 25 (1997), 2395-2407.

Department of Mathematics, Birzeit University, P.O.Box 14, West Bank, Palestine E-mail: msaleh@birzeit.edu

(Received November 25, 2002, revised November 30, 2003) 